\title{
Towards assessing online uncertainty for three-phase flow metering in the oil and gas industry
}

\author{
M. P. Henry, M. S. Tombs, and F. B. Zhou \\ University of Oxford, Oxford, UK \\ Correspondence to: M. P. Henry (manus.henry@eng.ox.ac.uk)
}

Received: 29 November 2013 - Revised: 19 March 2014 - Accepted: 21 March 2014 - Published: 22 April 2014

\begin{abstract}
A new three-phase (oil/water/gas) flow metering system has been developed for use in the oil and gas industries, based on Coriolis mass flow metering. To obtain certification for use in the Russian oil and gas industries, trials have taken place at the UK and Russian national flow laboratories, NEL in Glasgow and VNIIR in Kazan, respectively. The metrology of three-phase flow is complex, and the uncertainty of each measurement varies dynamically with the operating point, as well as the metering technology, and other aspects. To a limited extent this is reflected in the error limits allowed in national standards, which may vary with operating point. For example, the GOST standard allows errors in the oil flow rate of $\pm 6 \%$ for water cuts of less than $70 \%$, which is increased to $\pm 15 \%$ for water cuts between 70 and $95 \%$. The provision of online uncertainty for each measurement, for example in accordance with the British Standard BS-7986, would be highly desirable, allowing the user to observe in real time variations in measurement quality. This paper will discuss how an online uncertainty assessment could be implemented in the Coriolis meter-based system.
\end{abstract}

\section{Introduction}

A Coriolis mass flow meter (Fig. 1) consists of a vibrating flowtube through which the process fluid passes, and an electronic transmitter. The transmitter maintains flowtube vibration by sending a drive signal to one or more drivers, and performs measurement calculations based on signals from two sensors. The physics of the device dictates that Coriolis forces act along the measurement section between the two sensors, resulting in a phase difference between the sinusoidal sensor signals. This phase difference is essentially proportional to the mass flow rate of the fluid passing through the measurement section.

The frequency of oscillation of the flowtube varies with the density of the process fluid. The frequency value can be extracted from the sensor signals (for example by calculating the time delay between consecutive zero crossings) so that the process density can be obtained. The flowtube temperature is also monitored to enable compensation for variations in flowtube stiffness.

Coriolis meters are widely used throughout industry. The direct measurement of mass flow is often preferred over volumetric-based metering, for whereas the density and/or volume of a material may vary with temperature and/or pressure, mass remains unaffected. This is particularly important in the oil and gas industry, where energy content and hence product value is a function of mass.

The exploitation of new technology, such as audio quality analog-to-digital convertors and digital-to-analog convertors (ADCs and DACs), and field-programmable gate arrays (FPGAs), has facilitated the development of new capabilities for Coriolis meters, such as the ability to deal with multiphase flows. Multiphase flow introduces highly variable damping on the flowtube, up to three orders of magnitude higher than in single phase conditions, requiring agile and precise drive control, which only the latest technology can provide. In addition, the mass flow and density measurements generated under multiphase flow conditions are subject to large systematic and random errors, for which correction algorithms must be defined and implemented.

There is great interest within the oil and gas industry for exploiting the new Coriolis metering technology in upstream applications, where the process fluids are inherently multiphase. A Coriolis meter measuring two parameters - mass 


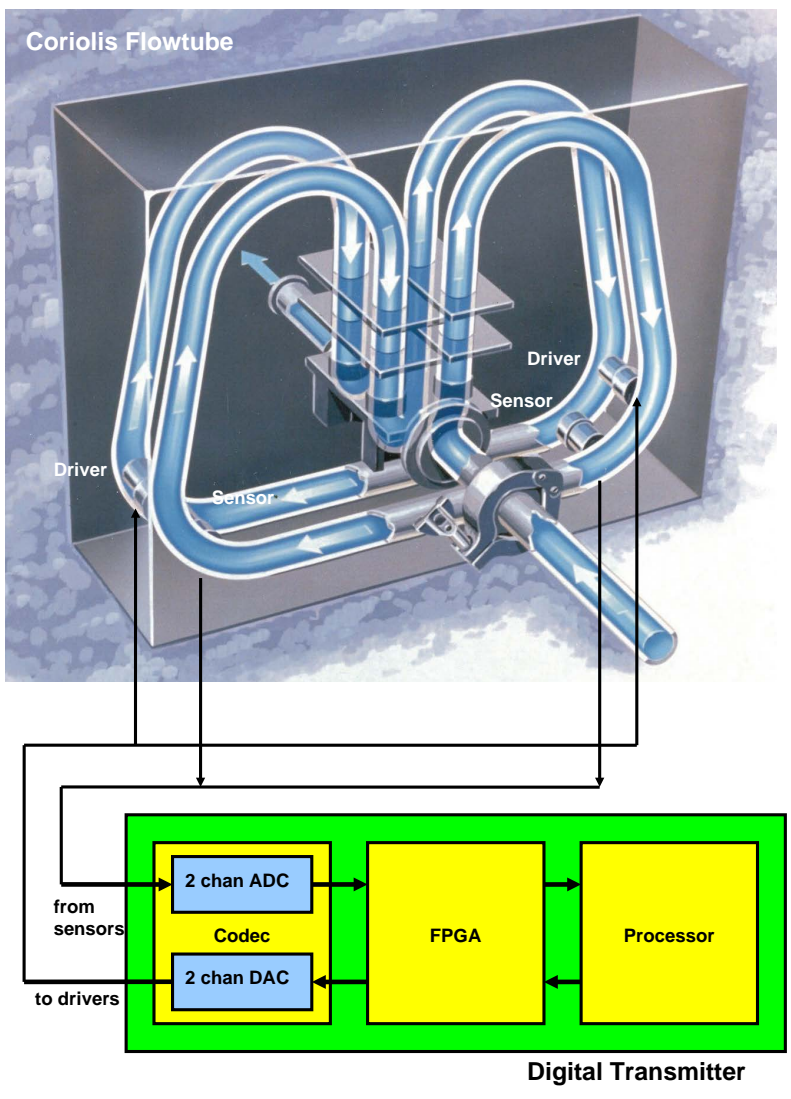

Figure 1. Coriolis mass flow meter: flowtube and digital transmitter.

flow and density - is theoretically able to resolve a twophase (liquid/gas) mixture. However, unless simplifying assumptions are made, a Coriolis meter cannot on its own resolve the general three-phase oil/water/gas mixture that characterises most oil well production. Including a third measurement, such as water cut (the proportion of water in the liquid mixture, typically scaled between 0 and $100 \%$ ), enables true three-phase metering to be achieved.

The term "Net Oil" is used in the upstream oil and gas industry to describe the oil flow rate within a three-phase or a liquid (oil/water) stream. A Net Oil \& Gas Skid (from here on referred to as the Skid) measures the oil and gas flow rates, and hence also the produced water, in a three-phase produced fluid.

The Skid (Henry et al., 2013) has been designed by the authors and their industrial partners as a replacement for threephase separator measurement systems conventionally used for well testing and production monitoring in the field. Figure 2 shows the design of the Skid. The pipework dimensions and internal diameter $(50 \mathrm{~mm})$ remain the same while a range of Coriolis meter inlet diameters from 15 to $50 \mathrm{~mm}$ can be fitted to match the flow rate of the wells to be monitored. The Skid has been successfully tested at the UK and Russian

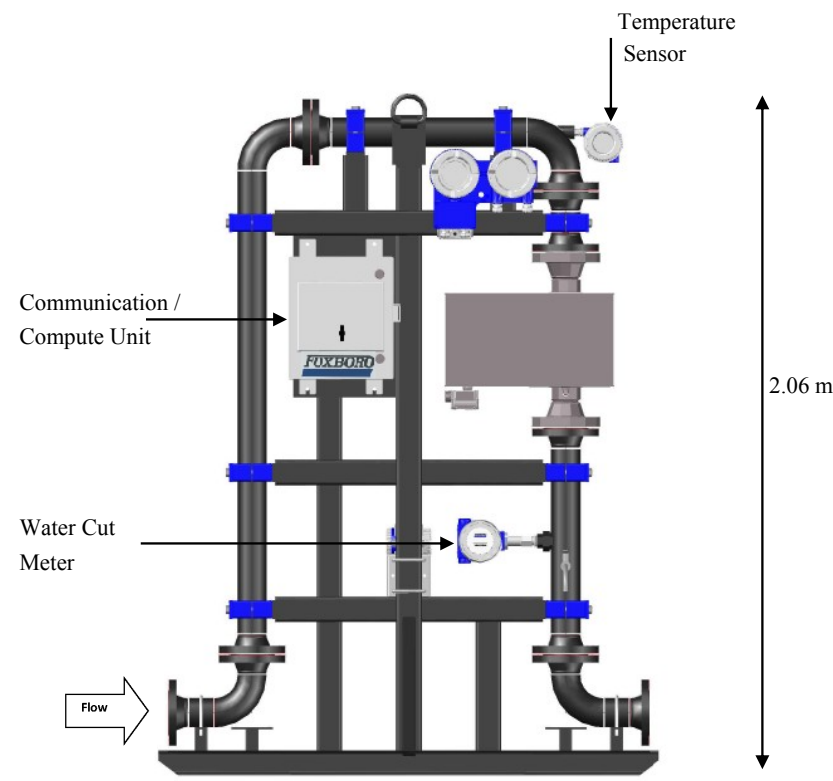

Figure 2. Net Oil and Gas Skid.

national flow laboratories, and is currently undergoing field trials.

The metrology of three-phase flow is complex, and in reality the uncertainty of each measurement varies dynamically with the operating point, as well as the metering technology, and other aspects. The need to accommodate variations in measurement quality at different three-phase operating points is acknowledged to a limited extent in some national standards (e.g. GOST, 2008). However, it is argued in this paper that a truly dynamic uncertainty analysis of the three-phase measurements would facilitate extending the range of operating conditions under which guaranteed measurement performance could be provided. It is further argued that the best approach to constructing a three-phase flow uncertainty analysis is through the use of Monte Carlo modelling.

After describing the design and performance of the Skid, this paper will discuss the steps needed to provide an online assessment of the uncertainty of the three-phase measurements, conforming to the SEVA concept, as specified in the British Standard BS-7986 (BSI, 2005), as well as the international standard known as the GUM - the Guide to the Expression of Uncertainty in Measurement (JCGM, 2008a).

\section{Net Oil \& Gas Skid}

The Skid (Fig. 2) is designed to condition the process fluid flow to minimise slip between gas and liquid via the rise and fall of the pipework, and by an integrated flow straightener in the horizontal top section. The Coriolis meter is positioned on the downward and outward leg of the Skid. Other instrumentation consists of a water cut meter and a pressure and temperature transmitter. The latter reads the pressure at the 


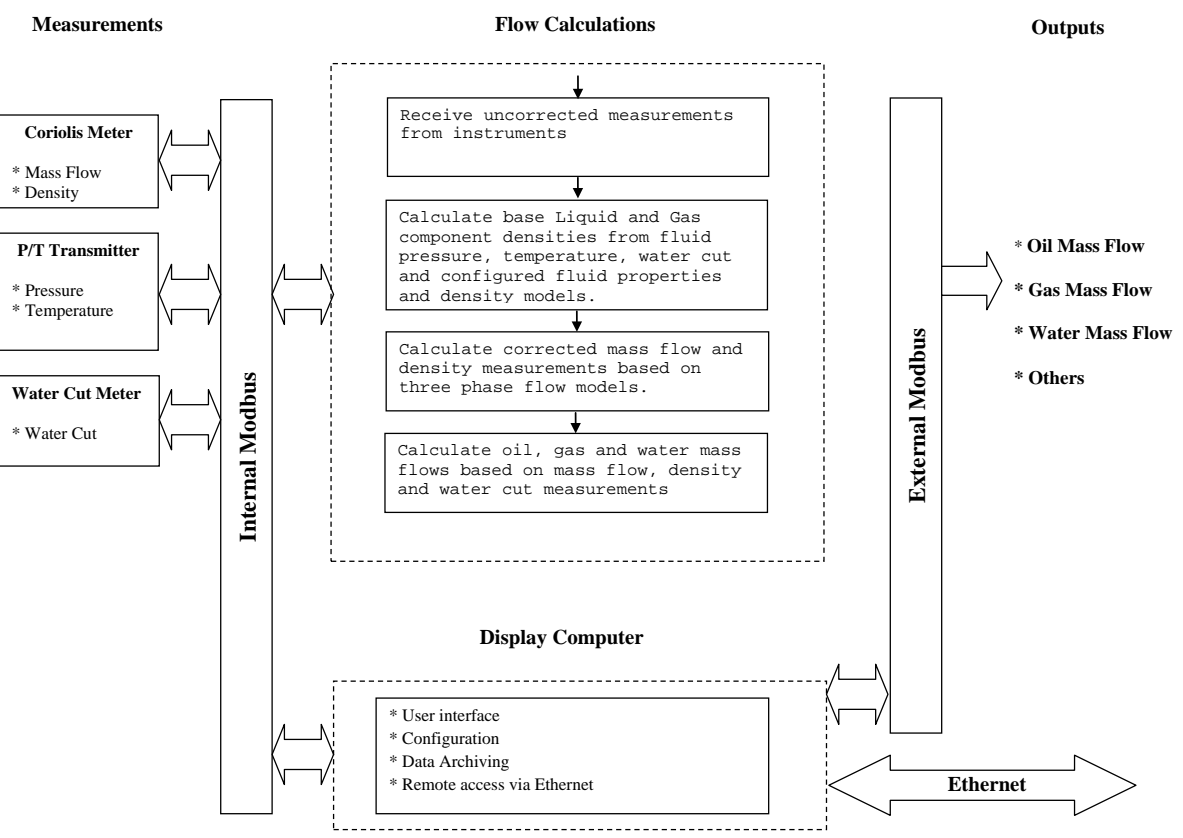

Figure 3. Hardware/software architecture of the Net Oil and Gas system.

inlet to the Coriolis meter and the temperature of an RTD (resistance temperature detector) sensor in a thermal well, positioned at the top of the Skid. The Communication/Compute Unit acts as a communication master for all the devices, using the Modbus industrial communications protocol, commonly used in the oil and gas industry. The Compute Unit performs three-phase flow measurement calculations based on the data received, provides a user interface (for providing, for example, gas and fluid density information) and also carries out data archiving. Real-time data is provided to the user's data acquisition system via a Modbus interface, with an update rate of 1 second.

The hardware/software architecture of the Skid is shown in Fig. 3. The Display Computer provides three communication interfaces: an internal Modbus for the Skid instrumentation, an external Modbus interface to provide measurement values to the user, and an Ethernet interface to enable remote configuration, monitoring and archival data retrieval. The Display Computer further provides a user interface to enable local configuration, data display, etc.

Figure 3 further shows an overview of the flow calculation algorithm. The uncorrected data from the instruments is gathered via the Modbus interface. Here, "uncorrected" refers to the effects of multi-phase flow: the mass flow, density and water cut readings are calculated based on their single-phase calibration characteristics. The liquid and gas densities are calculated based on the temperature, pressure and water cut readings and configuration parameters, based on data provided by the user. Corrections are applied to the Coriolis meter mass flow and density readings based on the three-phase flow measurement models. Finally, the oil, water and gas measurements are calculated from the corrected mass flow, density and water cut.

The corrections to the mass flow and density readings are implemented using neural networks, based on internally observed parameters. One important parameter is the density drop, i.e. the difference between the pure liquid density (for a particular water cut value) and the observed density of the gas/liquid mixture. For example, Fig. 4 shows a 3-D visualisation of the observed density drop error against the observed mass flow and density drop, keeping other parameter values constant (e.g. the water cut is $52 \%$ ). Here a zero density drop indicates no gas present and, as would be expected, results in no density error. Models based on laboratory experimental data are used to provide online corrections for the mass flow and density readings.

Once models have been constructed and implemented in real-time software, formal trials can be carried out to test the resulting performance, and to demonstrate compliance with oil industry standards. For example, the Russian Standard GOST R 8.165 (GOST, 2008) has the following key specifications:

- Total liquid flow accuracy requirement $\pm 2.5 \%$

- Total gas flow accuracy requirement $\pm 5.0 \%$

- Total oil flow accuracy requirement dependent upon water cut:

- For water cuts $<70 \%$, oil accuracy requirement $\pm 6.0 \%$

- For water cuts $>70 \%$ and $<95 \%$, oil accuracy requirement $\pm 15.0 \%$ 


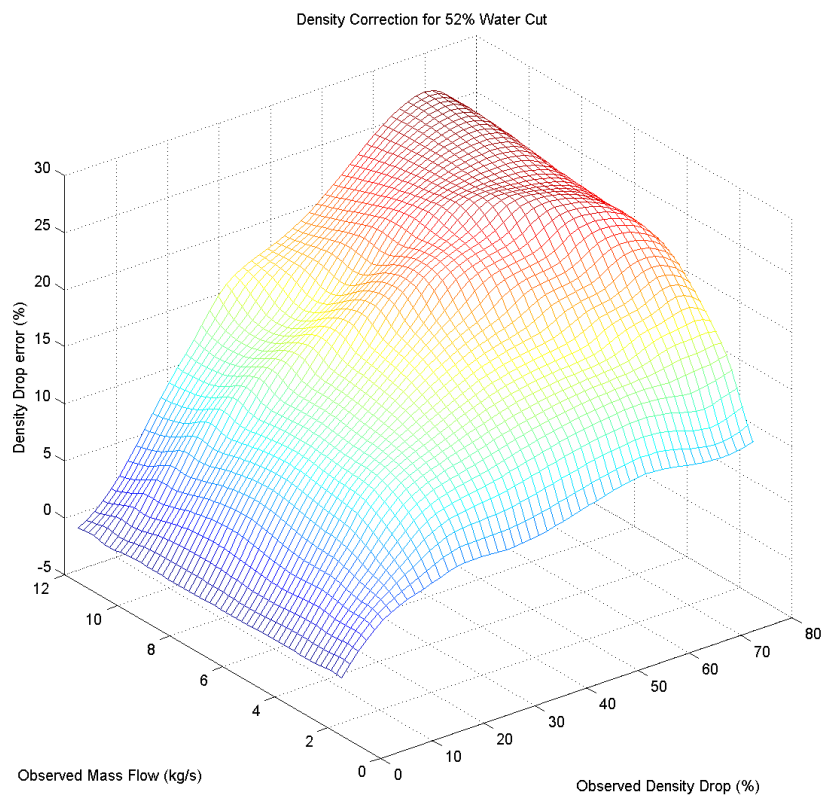

Figure 4. Density error induced by effects of three-phase flow.

- For water cuts $>95 \%$, no universal oil accuracy requirement is specified. Instead, the limit of the permissible relative error is to be specified in a measurement procedure to be approved and validated by the appropriate authorities.

These accuracy requirements are absolute, i.e. all test results must fall within these specifications. Trials have taken place on the Skid at the UK national flow laboratory, NEL, in Glasgow, and at the Russian national flow laboratory, VNIIR in Kazan. The resulting performance (Henry et al. 2013) matches the GOST requirements, and the Skid has been certified for use in Russia. For example, Fig. 5 shows the liquid mass flow errors from 75 formal trials at NEL, over the full range of water cuts, where the specified accuracy requirement is $\pm 2.5 \%$.

\section{Motivation for an online uncertainty analysis}

Typically, formal trials at laboratories are carried out under steady state conditions. For example, in Fig. 5, each test result is based on a five-minute trial where all reference conditions are kept constant. The advantage of testing at steady state is that it reduces the uncertainty of the reference flow rates so that the performance of the Skid can be accurately assessed at specific operating points.

In practice, a desired accuracy performance can only be achieved over a limited range of conditions. For example, the maximum total liquid flow rate achievable through the Skid is likely to be determined by pressure drop considerations; conversely the minimum total liquid flow rate is likely to be constrained by the accuracy performance of the Skid at

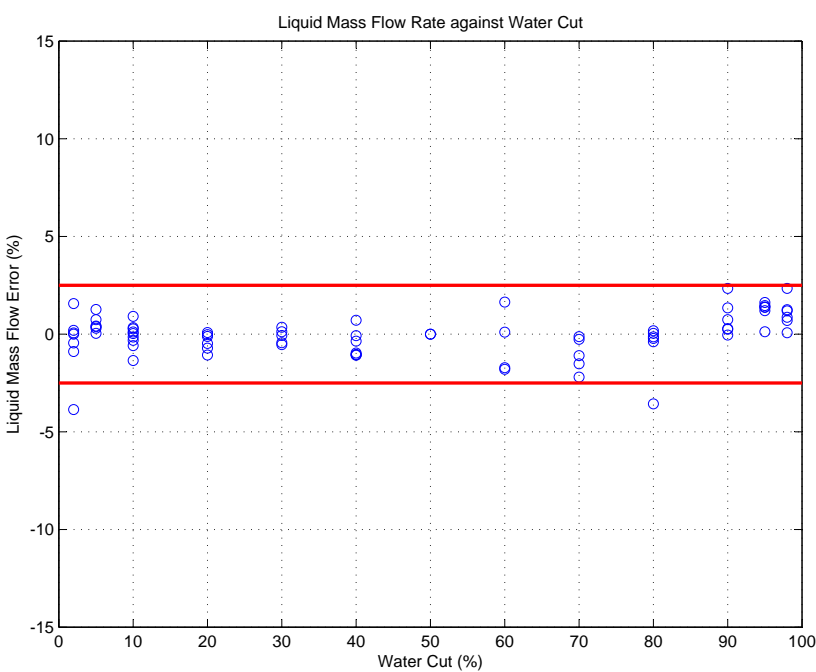

Figure 5. Total liquid mass flow rate errors against water cut. The target limit is $\pm 2.5 \%$ (red boundary).

low flow. With three-phase flow, there are many dimensions to consider in specifying the operating envelope for acceptable measurement uncertainty. For example, as the water cut increases towards $100 \%$, it becomes increasingly difficult to measure the absolute oil flow rate to within $\pm 6.0 \%$; in this case the GOST standard varies the oil flow rate accuracy requirement with the water cut, as discussed above, but no such provision is made for the gas flow measurement, which is required to be accurate to within $5 \%$ in all cases. As the gas volume fraction (GVF) tends to zero, it becomes increasingly difficult to meet this requirement.

For example, consider a mixture of pure water and gas, where the water density is taken as $1000 \mathrm{~kg} \mathrm{~m}^{-3}$, the gas density at line temperature and pressure is $5 \mathrm{~kg} \mathrm{~m}^{-3}$, and the GVF is $5 \%$. Then in every cubic metre of gas/liquid mixture, there are $950 \mathrm{~kg}$ of water, and only $250 \mathrm{~g}$ of gas; the GOST standard requires the latter to be measured to within $\pm 12.5 \mathrm{~g}$. To achieve this resolution for gas dispersed within $950 \mathrm{~kg}$ of water is extremely challenging, although this performance was successfully achieved in trials at NEL (Henry et al., 2013).

Testing performance with static flow conditions in laboratories can thus be used to set limits on the range of parameters over which the Skid can deliver the required accuracy performance. In practice, the accuracy of each of the oil, water and gas flow measurements will vary dynamically with the operating point (e.g. water cut, GVF and liquid mass flow rate), as well as other conditions (e.g. process noise).

Furthermore, real oil and gas wells often exhibit dynamic behaviour. For example, Fig. 6 shows data from a field trial in Russia over the course of a three-hour test. The upper graph shows the proportion by volume of free gas, oil and water in the produced fluid, while the lower graph shows the absolute volumetric flow rates. Here the well flow rate and composition show significant dynamic variation in water cut, GVF, 

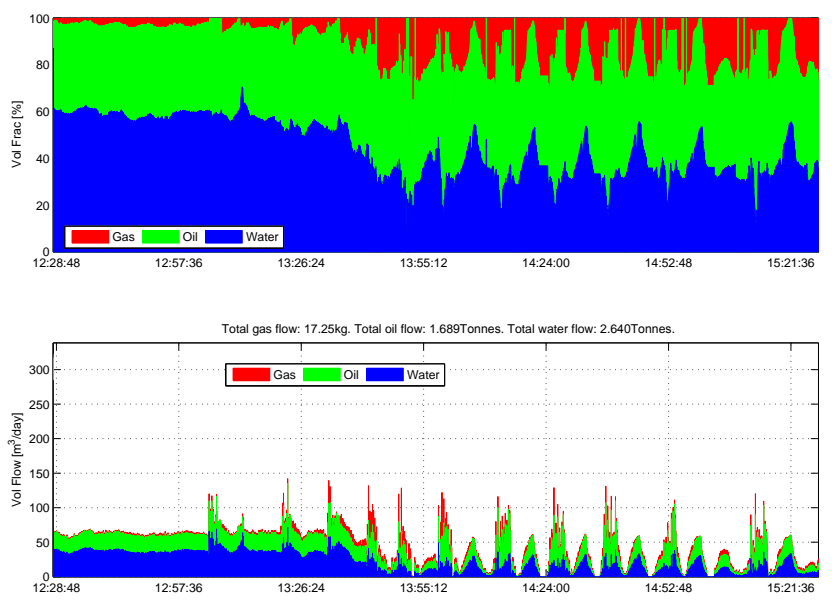

Figure 6. Oil, water and gas measurements from a Russian field trial.

and liquid flow rate. One major advantage of the Skid over conventional separator technology is that it provides dynamic measurements, as opposed to simple totalised flows over a period of several hours. Data on the dynamics of flow are potentially useful to reservoir engineers for understanding the evolving state of the oilfield.

Conventionally, it is assumed that as long as the Skid operating conditions fall within the specification of the certification standard (e.g. GOST) throughout the entire well test period, then the measurement accuracy can be considered to be within the specified limits (e.g. $5 \%$ for gas flow).

A more pragmatic and flexible approach would be to assert that, for a particular well test, as long as the operating conditions averaged over the duration of the test fall within the specification of the certification standard, then nominal accuracy can be assumed.

An alternative approach would be to provide a dynamic uncertainty analysis for each measurement value, as a function of the operating conditions, process noise and other influencing factors. With this approach, the overall uncertainty of each measurement is estimated, based upon its dynamic behaviour over the course of the well test period.

In particular, this approach might facilitate the demonstration of acceptable levels of uncertainty over wider ranges of operating conditions than for a purely static analysis. For example, if the liquid flow rate drops below the threshold for acceptable accuracy based on a static analysis, a dynamic uncertainty analysis may demonstrate that the contribution of this low flow to the overall uncertainty of the entire test period may be small, and that the overall well test total flow remains within specification.

Thus, developing a dynamic uncertainty analysis for the Skid may be able to demonstrate acceptable uncertainty performance over a wider range of operating conditions than is possible using static, laboratory-based verification.

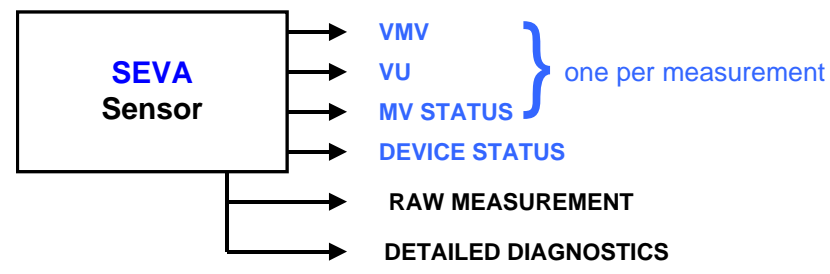

Figure 7. A SEVA sensor generating measurement and validity data.

\section{Online uncertainty and SEVA}

The Sensor Validation (SEVA) concept (Henry and Clarke, 1993) proposes a model of how a "self-validating" or SEVA sensor should behave, assuming the availability of internal computing power for self-diagnostics, and of digital communications to convey measurement and diagnostic data. This model has been incorporated into the British Standard BS7986 (BSI, 2005). Note that a similar concept, "metrological diagnostic self-regulation", has been developed independently in Russia, and this has been incorporated into national standards (GOST, 2009, 2011). A generic set of metrics are proposed for describing measurement quality (see Fig. 7). For each measurement, three parameters are generated:

- The Validated Measurement Value (VMV). This is the best estimate of the true quantity value of the measurand, calculated using an automated measurement procedure. Where diagnostic information indicates a known fault, the measurement procedure is adjusted to compensate for the fault. Typically such diagnostic information will be derived from one or more reference values (ISO, 1994; BIPM 2012) internal to the sensor, the Validated Uncertainty (VU). This is the measurement uncertainty, or probably error, of the VMV. For example, if the VMV is $4.31 \mathrm{~kg} \mathrm{~s}^{-1}$, and the VU is $0.05 \mathrm{~kg} \mathrm{~s}^{-1}$, then the sensor is claiming that the true measurement value lies between 4.26 and $4.36 \mathrm{~kg} \mathrm{~s}^{-1}$ with the stated level of coverage (typically $k=2,95 \%$ probability).

- The Measurement Value Status (MV Status). Given the requirement to provide a measurement, even when a fault has occurred, the MV Status indicates the generic fault state under which the current measurement value has been calculated.

For the purposes of this work, the most important aspect of the SEVA scheme is the generation of the Validated Uncertainty, a dynamic assessment of the uncertainty associated with each measurement value provided by the sensor. In the case of a complex instrument such as a Coriolis meter, the uncertainty of each measurement (e.g. the mass flow and density) is calculated separately within the instrument, and will vary dynamically with operating point, process noise and other parameters. Online uncertainty can be used for a 
variety of purposes, such as deciding on control system behaviour (e.g. whether to accept or reject the quality of the measurement value for the purposes of taking control decisions). Where measurements are combined (for example in forming mass balances or other higher-level calculations), the SEVA scheme proposes the provision of a higher-level uncertainty analysis, where the dynamic uncertainty of the input measurements are used in the calculation of the uncertainty of the resulting measurement. Consistency checking between redundant SEVA measurements has also been developed (Duta and Henry, 2005).

Here, it is assumed that dynamic assessments of the uncertainty of each measurement from the Coriolis meter, water cut meter and other sensors are available, and that these will be used to generate a corresponding online uncertainty assessment of the three-phase measurements of gas, water and oil flow.

\section{Towards assessing online uncertainty for three-phase flow metering}

In the Guide to the Expression of Uncertainty in Measurement or GUM (JCGM, 2008a), a number of techniques are described for calculating the uncertainty of an output variable from the values and uncertainties of input variables. In the case of a simple analytical relationship between inputs and output, formulaic expressions can be used. In more complex cases, where for example there may be a correlation between input variables and/or the functional relationship is not readily expressed algebraically, Monte Carlo modelling (MCM) is proposed as an alternative technique (JCGM, 2008b). Given the complexity of the three-phase flow calculations, which includes neural net models, MCM is proposed as the most appropriate means of assessing output uncertainty (Fig. 8) for the Skid.

In outline, with MCM the measurement calculation is carried out multiple times, where in each case the input variables are randomly selected based on their respective probability distributions. With a sufficient number of repeat measurements, it is possible to estimate the probability distribution of each output variable, and thereby to calculate a mean and coverage interval or uncertainty.

The GUM is primarily intended for static, offline analyses. In Sect. 7 of Supplement 1 (JCGM, 2008b), where the number of Monte Carlo trials $M$ is discussed, it is suggested that one million simulations might be appropriate to ensure a good approximation of the distribution of the output variable $Y$. This is clearly unlikely to be feasible in an online Skid with a $1 \mathrm{~s}$ update rate. However, the following text appears applicable:

"If the model is complicated, ..., because of large computing times it may not be possible to use a sufficiently large value of $M$ to obtain adequate distributional knowledge of the output quantity. In such a case an approximate approach

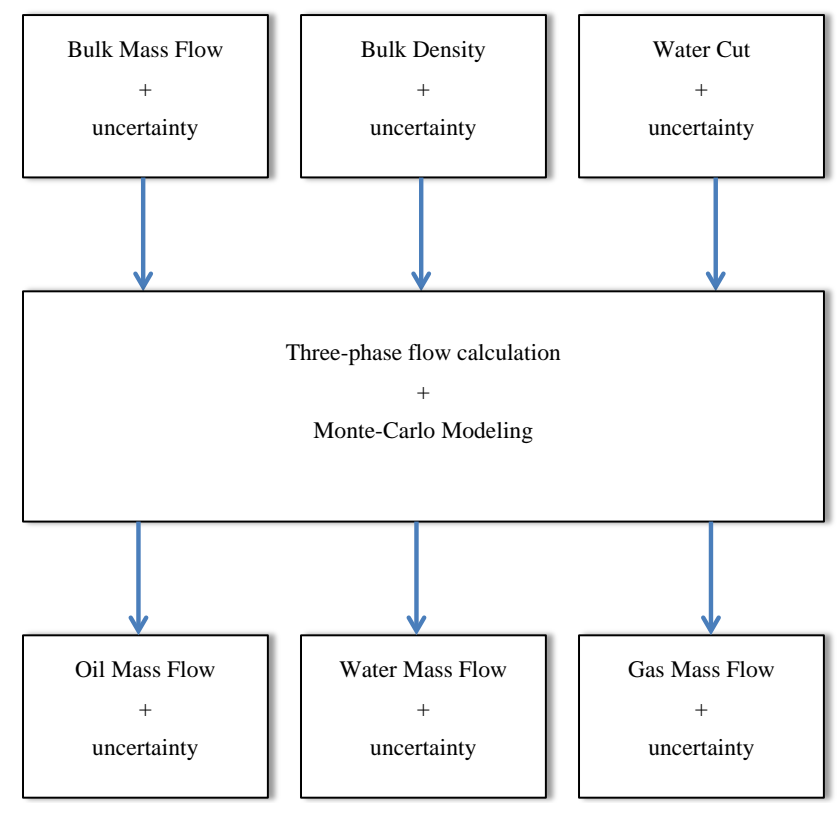

Figure 8. Monte Carlo modelling to estimate output uncertainties.

would be to regard [the distribution of $Y$ ] as Gaussian (as in the GUM) and proceed as follows. A relatively small value of $M, 50$ or 100 , for example, would be used. The average and standard deviation of the resulting $M$ model values of $Y$ would be taken as its [mean and uncertainty] respectively."

The proposed approach will be implemented as follows:

- At the start of each new calculation period, mass flow, density, water cut, pressure, and temperature measurements are collected from the Skid instrumentation.

- Estimates of the uncertainties of each of these measurements are obtained either from the instruments themselves, or in the display computer.

- Simple Gaussian distributions can be assumed. The only likely correlations are between the mass flow and density measurements - all others can be assumed to be independent.

- Monte Carlo modelling simulation will entail performing 50-100 three-phase measurement calculations where the input parameters are randomly selected from their assumed Gaussian distributions.

- The resulting oil, water and gas mass flow rates are assumed Gaussian, so that the best estimate and uncertainty of each flow rate can be calculated from the MCM results.

- The totalised flow and its uncertainty are updated for each fluid type. 
It will be challenging to validate the model especially based on only $50-100$ points and to prove that this is representative. However, even with only 50-100 MCM calculations per measurement update, this approach will require a substantial increase in the computing power resources for the Skid if it is to be implemented in real time. A first step will be to implement an offline simulation of the MCM-based uncertainty analysis. Offline case studies based on field trial data will be used to evaluate the utility of online uncertainty analysis. The challenges of real-time implementation will be addressed once the benefits are demonstrated in simulation.

Acknowledgements. The authors thank Invensys Process Systems for funding this research, and our two reviewers for their helpful and insightful comments.

Edited by: R. Tutsch

Reviewed by: two anonymous referees

\section{References}

BIPM: BIPM, JCGM 200, Vocabulary of Metrology - Basic and General Concepts and Associated Terms, 3rd Edn., 2008 version with minor corrections, 2012.

BSI: BS7986:2005, Specification for data quality metrics for industrial measurement and control systems, British Standards Institute, 2005.

Duta, M. D. and Henry, M. P.: The fusion of redundant SEVA sensors, IEEE T. Contr. Syst. T., 13, 173-184, 2005.
GOST: R 8.615, Amended 2008: State system for ensuring uniformity of measurements. Measurement of quantity of oil and petroleum gas extracted from subsoil. General metrological and technical requirements, Federal Agency for technical regulation and metrology, 2008 (in Russian).

GOST: R 8.674-2009, State system for ensuring the uniformity of measurements. Intelligent Sensors and Intelligent Measuring Systems. Basic Terms and Definitions, 2009 (in Russian).

GOST: R 8.734-2011, State system for ensuring the uniformity of measurements. Intelligent Sensors and Intelligent Measuring Systems. Methods of metrological self-checking, 2011 (in Russian).

Henry, M. P. and Clarke, D. W.: The Self-Validating Sensor: Rationale, Definitions and Examples, Control Eng. Pract., 1, 585-610, 1993.

Henry, M. P., Tombs, M. S., Zamora, M. E., and Zhou, F. B.: Coriolis Mass Flow Metering for Three-Phase Flow, Flow Meas. Instrum., 30, 112-122, 2013.

ISO: 5725-1:1994, Accuracy (trueness and precision) of measurement methods and results - Part 1: General principles and definitions, 1994.

JCGM: JCGM 100:2008, Evaluation of measurement data Guide to the expression of uncertainty in measurement, http://www.bipm.org/utils/common/documents/jcgm/JCGM_ 100_2008_E.pdf (last access: 17 April 2014), 2008a.

JCGM: JCGM 101:2008, Evaluation of measurement data — Supplement 1 to the "Guide to the expression of uncertainty in measurement" - Propagation of distributions using a Monte Carlo method, http://www.bipm.org/utils/common/documents/ jcgm/JCGM_101_2008_E.pdf (last access: 17 April 2014), 2008b. 